\title{
Nonlinear Observer-Based Tracking Control for Induction Motors
}

\author{
Jung Hua Yang ${ }^{1}$, Wen Hai Yu ${ }^{1}$, and Li Chen $F^{1,2}$
}

1. Department of Electrical Engineeging

2. Department of Computer Science \& Information Engineering

National Taiwan University, Taipei, Taiwan, R.O.C.

\author{
Abstract \\ stage design and technique is applied to construct an observer-based controller \\ lators. Simulation results have verified the effective performance of the controller. \\ the other is to perform the function of pulse width modulation (PWM) and the \\ generation of gating pulses. Satisfactory experimental studies are also shown in \\ this paper. \\ Nomenclature : \\ $v_{a s}, v_{b s}, v_{c s}\left(v_{a r}, v_{b r}, v_{c r}\right):$ stator (rotor) phase voltages \\ $i_{a,} i_{b o} i_{c o s}\left(i_{a r} i_{b r} i_{c r}\right):$ stator (rotor) phase currents \\ $v_{d o}\left(v_{q s}\right) ; d$-axis (q-axis) stator voltage \\ $i_{d s}\left(i_{q s}\right): d$-axis (q-axis) stator current \\ $\Phi_{d r}\left(\Phi_{q r}\right):$ d-axis (q-axis) rotor flux \\ $\theta_{s}:$ position of rotor flux vector \\ $\theta_{r}$ : rotor angle \\ $\theta_{s i}:$ slip angle \\ $\omega_{s}:$ stator angular frequency \\ $\omega_{r}:$ rotor angular speed \\ $\omega_{s t}:$ slip angular speed \\ $L_{l s}\left(L_{l r}\right):$ leakage inductances of stator (rotor) windings \\ $L_{m s}\left(L_{m r}\right):$ magnetizing inductances of stator (rotor) windings \\ $L_{a r}$ : mutual inductances between stator and rotor windings \\ $R_{s}\left(R_{r}\right)$ : stator (rotor) resistance \\ $L_{s}\left(L_{r}\right):$ stator (rotor) self-inductance \\ $M:$ stator/rotor mutual inductance \\ $p$ : number of pole pairs \\ $\mathrm{J}$ : rotor inertia \\ D : damping coefficient \\ $K_{T}:$ torque constant $\left(=3 p M / 2 L_{r}\right)$ \\ $T_{L}$ : disturbance torque \\ $\sigma$ : leakage coefficient $\left(=1-M^{2} / L_{s} L_{r}\right)$ \\ $c: 1 / \sigma L_{s}$ \\ $a_{1}: c\left(R_{s}+M^{2} R_{r} / L_{r}^{2}\right)$ \\ $a_{2}: c M R_{r} / L_{r}^{2}$ \\ $a_{3}: p c M / L_{r}$ \\ $a_{4}: R_{r} / L_{r}$ \\ $a_{5}: M R_{r} / L_{r}$
}

In this paper, we propose a nonlinear observer-based controller for induction motors. Via the use of skew-symmetrical property of induction motors, a twofor velocity and position tracking control. To demonstrate the effectiveness of the proposed scheme, the scheme is applied to the tracking control of robot manipu-

To implement the proposed scheme for experiment, a voltage-control type of drive system has been set up, which employs two personal computers, PC 486 and PC 286. One is adopted to perform the calculation of the control law and

\section{Introduction}

The induction motor has a wide variety of applications as the electromechanical actuator because of its ruggedness, low maintenance, and low cost. Advances in power electronics and microprocessor technology make it feasible to use the induction motors in place of $d c$ and synchronous motors in a wide range of servo applications. However it is very difficult to achieve high performance with an induction motor, which is caused by the nonlinear coupling terms of the dynamics comparing the electrical part and the mechanical torque part.

In a separate-excitation dc motor, the electromechanical arrangement provides decoupling between magnetic effects in the stator and in the rotor windings. This decoupling is reflected in the torque dynamical equation. The $\mathrm{dc}$ motor can be linearly operated simply by keeping the stator current constant.

However, for an induction motor, the stator windings produce an associated flux, and indirectly create a rotor flux as well. Angular separation between the fluxes results from the time delay inherent in the rotor circuit. As the stator current is the only mechanism for torque generation, the stator and rotor effects are tightly coupled. Thus, the motor machine cannot be operated as a linear device unless some kind of nonlinear feedback is applied.
To overcome the problems mentioned above in practical implementations, the field orientation control [12] is a widely used approach. This method uses a nonlinear feedback to obtain the approximate decoupling between the stator and rotor and cause the dynamics of an induction motor to behave like those of a separateexcitation dc motor. When the full-state feedback is used, it is called direct field orientation control $[8,9]$. But it requires accurate flux sensors which are expensive and suffer drawbacks such as temperature sensitivity and its induced changes to the motor structure. Another approach is to use observers for flux in place of the previous sensors. This method is called indirect field orientation control. Its stability, robustness, and related control properities are, however, not fully studied yet, although the field orientation control performs well in practice.

In the past few years, the advances in nonlinear control theroy have had a notable impacts on the field of induction motor control. Input-output decoupling control was presented in [4] and [3] using geometric techniques. In [4], a simplified model is used, i.e., only the electromagnetic part is considered and the speed is assumed to be a slowly varying parameter. Exact decoupling in the control of electric torque and flux amplitude using the amplitude and frequency of the voltage supply as inputs are achieved by a static state feedback. On the other hand, the results in $[21,22]$ are based on the adaptive control theories of linear systems so that linearized models of induction motors are used for controller design and stability analysis. Along the same line, the work in [18] proposed an adaptive tracking control scheme based on the nonlinear model. Moreover, Marino $[5,6]$ proposed a direct adaptive controller for speed regulation in which the motor model is input-output decoupled by a feedback-linearizing controller but with the load torque and rotor resistance being adapted in time. The results of that is the ensurance of asymptotic convergence of the system states and the estimated parameters.

In aforementioned schemes $[4,5,6,18]$, the full states must be available. So practical implementaions will require the flux obeservers, and hence a good deal of research effort has been directed towards the design of nonlinear controllers that employ flux observers. In $[5,6]$, the controller was combined with the flux observer [10]. However, no proof of stability was given for the resulting closed-loop system.

In [3], the observer-based adaptive controller was designed and the closed-loop stability was proved under several restrictive assumptions. In [15], closed-loop stability is also proved by using design technique for nonlinear feedback control [16]. But the singularity of the control laws remains as in $[3,4,5,6,18,15]$. The globally stable controller for regulating torque and flux amplitudes was presented in [17]. Using the skew-symmetric properties of the model of induction motors, a controller without singularity is designed by using a fourth-order observer, but only torque tracking and flux regulation are achieved.

In this paper, we propose a nonlinear observer-based controller for induction motors. Via the use of skew-symmetric properties of the model [17], the twostage design technique [18] is applied to construct an observer-based controller for velocity and position tracking control. To demonstrate the effectiveness of the proposed scheme, an application to the tracking control of robot manipulators is presented. Simulation results show that the tracking is satisfactory and both the input voltage signals as well as the current signals remain within the acceptable limits.

In order to implement the proposed scheme for experiment, a voltage-control type of drive system $[13,14]$ has been set up, which employs two personal comput ers, namely, PC 486 and PC 286. One is adopted to perform the calculation of the control laws and the other is to perform the function of pulse width modulation (PWM) and generation of gating pulses.

The layout of this paper is as follows. The nonlinear model of the induction motors is shown in section 2. Such a model is then rearranged for the controller design, in which torque load is taken into more realistic considerations. In section 3 , the controller is proposed and the main results of this paper are derived as well. The simulation results are shown in section 4 , and experimental setup as well as the results are presented in section 5. Finally, the conclusions are provided in section 6 . 


\section{Model of Induction Motors}

With the notations in [1] [3], the dynamics of a balanced 3-phase Y-connected that has symmetric, and linear magnetic circuits can be described as a fifth-order state-space dynamical system. Let the motor have p poles, then, after a coordinate transform onto the $d-q$ coordinate frame rotating synchronously with an angular speed $w_{2}$, the dynamical system can be represented as follows:

$$
\begin{aligned}
& i_{d \rho}=-a_{1} i_{d s}+\omega_{,} i_{q \rho}+a_{2} \Phi_{d r}+a_{3} \omega_{r} \Phi_{q r}+c V_{d r} \\
& i_{q \rho}=-\omega_{q} i_{d e}-a_{1} i_{q,}-a_{3} \omega_{r} \Phi_{d r}+a_{2} \Phi_{q r}+c V_{q} \text {, } \\
& \dot{\Phi}_{d r}=-a_{4} \Phi_{d r}+a_{5} i_{d \rho}+\left(\omega_{c}-p \omega_{r}\right) \Phi_{q r} \\
& \dot{\Phi}_{q r}=-a_{4} \Phi_{q r}+a_{5} i_{q s}-\left(\omega_{s}-p \omega_{r}\right) \Phi_{d r} \\
& \dot{\omega}_{r}=\left(-D_{\omega_{r}}+T_{e}-T_{L}\right) / J \\
& T_{c}=k_{T}\left(\Phi_{d r} i_{q o}-\Phi_{q r} i_{d e}\right)
\end{aligned}
$$

where $V_{d e}, V_{q s}, \omega_{\text {e }}$ are control inputs and $T_{c}$ is the generated torque. The definitions of the notations and symbols are given in the Nomenclature.

It can be seen shortly that the dynamical model described by equations (1)(6) possesses a skew-symmetric property, which will be used in the design of the controller. In order to manifest this property, we first rearrange the dynamical equations by using more compact notations as follows.

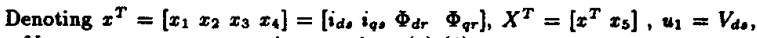
$u_{2}=V_{q s}, u_{3}=\omega_{s}$, we can rewrite equations $(1)-(6)$ as

$$
\begin{aligned}
& \dot{x_{1}}=-a_{1} x_{1}+u_{3} x_{2}+a_{2} x_{3}+a_{3} x_{5} x_{4}+c u_{1} \\
& \dot{x_{2}}=-u_{3} x_{1}-a_{1} x_{2}-a_{3} x_{5} x_{3}+a_{2} x_{4}+c u_{2} \\
& \dot{x_{3}}=-a_{1} x_{3}+a_{5} x_{1}+\left(u_{3}-p x_{5}\right) x_{4} \\
& \dot{x_{4}}=-a_{4} x_{4}+a_{5} x_{2}-\left(u_{3}-p x_{5}\right) x_{3} \\
& \dot{x_{3}}=\left(-D x_{3}+T_{c}-T_{2}\right) / J \\
& T_{6}=k_{T}\left(x_{3} x_{2}-x_{4} x_{1}\right)
\end{aligned}
$$

Note that the equations (7)-(10) can be further rewritten in a more compact symbolic form as

$$
\dot{x}=A x+B u
$$

where

with

$$
A=A_{0}+A_{1}+A_{2}, u^{T}=\left[\begin{array}{ll}
u_{1} & u_{2}
\end{array}\right],
$$

$$
\begin{aligned}
A_{0} \triangleq \operatorname{diag}\left[-a_{1},-a_{1},-a_{4},-a_{4}\right], \\
A_{1} \triangleq\left[\begin{array}{cccc}
0 & u_{3} & -a_{3} & 0 \\
-u_{3} & 0 & 0 & -a_{5} \\
a_{5} & 0 & 0 & \left(u_{3}-p x_{5}\right) \\
0 & a_{5} & -\left(u_{3}-p x_{5}\right) & 0
\end{array}\right], \\
A_{2} \triangleq\left[\begin{array}{cccc}
0 & 0 & \left(a_{2}+a_{3}\right) & p a_{3} x_{5} \\
0 & 0 & -p a_{3} x_{5} & \left(a_{2}+a_{5}\right) \\
0 & 0 & 0 & 0 \\
0 & 0 & 0 & 0
\end{array}\right],
\end{aligned}
$$

and

$$
B \triangleq\left[\begin{array}{ll}
c & 0 \\
0 & c \\
0 & 0 \\
0 & 0
\end{array}\right]
$$

It should be noted that $A_{1}$ is a skew-symmetric matrix, i.e., $A_{1}{ }^{T}=-A_{1}$.

In the following, we assume that the torque load is a known function of the rotor speed. This assumption is more realistic than the one with constant load $[15,17,18]$. It is well-known that the bearings and lots of viscous forces vary linearly with the speed, while the large-scale fluid systems such as pumps and fans have loads that typically vary as the square of the speed [19]. Hence, the torque load is assumed to be in the form of

$$
T_{2}=\mu_{0}+\mu_{1} x_{5}+\mu_{2} x_{5}^{2}
$$

where $\mu_{0}, \mu_{1}, \mu_{2}$ are some known constants.

\section{Control of Induction Motors}

Given the dynamic model of an induction motor described in the previous section, the control problem is stated subjected to some prior sssumptions about the system in the following.

Assumptions :

(A1) The motor parameters are known in advance.

(A2) Rotor speed $x_{3}$ and stator currents $x_{1}, x_{2}$ are measurable.

(A3) The desired rotor speed $\omega_{d} \in C^{2}$, i.e., $\omega_{d}^{(i)}$, the $i$-th derivative of $\omega_{d}, i=1,2$, exist and are continuous.

\section{Control Objective}

Given a desired rotor speed tracjectory $\omega_{d}(t)$, determine the control inputs $\omega_{1}, w_{2}, w_{3}$ such that the rotor speed $\omega_{r}$ can track the desired tracjectory exponentially in time $t$, i.e.

$$
x_{s} \rightarrow x_{s d}, \quad \text { as } t \rightarrow \infty \text {. }
$$

assuming that the signals $x_{1}, x_{2}$, and $x_{5}$ are available.

Futhermore, the rotor fluxes are also regulated according to the desired commands, i.e.,

$$
\begin{array}{ll}
\Phi_{d r} \rightarrow \beta, & \text { as } t \rightarrow \infty . \\
\Phi_{q r} \rightarrow 0, & \text { as } t \rightarrow \infty .
\end{array}
$$

In the following, the proposed controller is motivated by the two-stage analysis first presented in [18]. The model of an induction motor can be viewed as a cascade of two subsystem shown in Fig.1, or in other words a two-stage system.

\subsection{Control Strategy}

\subsubsection{The First Stage}

Referring to equation (11), i.e.

$$
J_{\dot{x}_{3}}+D x_{s}+T_{L}=T_{e},
$$

the output signal in this stage is $\omega_{r}$ and the generated torque $T_{e}$ is viewed as the input. If we want the output signal $x_{b}$ to track $x_{5 d}$, a sufficient condition is to have the input signal $T_{e}$ be equal to the desired torque $T_{d}$ as follows :

$$
T_{d}=J \dot{x}_{3 d}+k_{1} x_{s d}+\mu_{0}+\left(\mu_{1}+D-k_{1}\right) x_{s}+\mu_{2} x_{5}^{2},
$$

where $T_{L}$ is replaced by equation (14). Let $e_{3} \triangleq x_{3}-x_{s d}$, we then have

$$
J \dot{e}_{s}+k_{1} e_{5}=T_{e}-T_{d}
$$

Since $T_{*}$ is not the actual exogenous input, the right hand side of (16) can not be made identically zero.

\subsubsection{The Second Stage}

In this stage, we just take the equations (7)-(10) into consideration. Here, the generated torque $T_{c}$ is viewed as the output, and the input signals are $u_{1}, u_{2}$, and *3. It is easy to show that as $x_{4}=x_{4 d}=0, x_{3}=x_{3 d}=\beta, x_{2}=x_{2 d}=\frac{T_{1}}{k_{T} \beta}$, the generated torque will coincide with $T_{d}$ from (12).

Let $e=x-x_{d}$, where $x_{d}^{T}=\left[x_{1 d}, x_{2 d}, x_{3 d}, x_{4 d}\right], z^{T}=\left[x_{3} x_{4}\right], \hat{z}^{T}=\left[\hat{x}_{3} \hat{x}_{4}\right]$, so that $\tilde{z}^{T}=z^{T}-\hat{z}^{T}=\left[\hat{x}_{3}, \hat{x}_{4}\right]=\left[x_{3}-\hat{x}_{3} x_{4}-\hat{x}_{4}\right]$, where $\hat{x}_{3}$, $\hat{x}_{4}$ are the estimates of the flux states $x_{3}, x_{4}$. From equation (13), we have

$$
\dot{e}=\left(A_{0}+A_{1}+A_{2}\right) e-\dot{x}_{d}+\left(A_{0}+A_{1}+A_{2}\right) x_{d}+B u
$$

which motivates us to design the control inputs $u_{1}, u_{2}, u_{3}$, and $x_{1 d}$ as :

$$
\begin{aligned}
v_{1}= & {\left[\hat{x}_{1 d}+a_{1} x_{1 d}-u_{3} x_{2 d}+a_{5} x_{3 d}-\left(a_{2}+a_{5}\right) x_{3 d}\right.} \\
& -\left(a_{2}+a_{3}\right)\left(\hat{x}_{3}-x_{3 d}\right)-a_{3} x_{5}\left(\hat{x}_{4}-x_{4 d}\right) \\
& \left.-k_{2}\left(x_{1}-x_{1 d}\right)+v_{1}\right] / c \\
u_{3}= & {\left[\hat{x}_{2 d}^{2}+a_{1} x_{2 d}+u_{3} x_{1 d}+a_{3} x_{5} x_{3 d}\right.} \\
& +a_{3} x_{5}\left(\hat{x}_{3}-x_{3 d}\right)-\left(a_{2}+a_{5}\right)\left(\hat{x}_{4}-x_{1 d}\right) \\
& \left.-k_{3}\left(x_{2}-x_{2 d}\right)+v_{2}\right] / c \\
u_{3}= & p x_{3}+\frac{a_{5}}{\beta} x_{2 d}-\frac{1}{\beta} v_{4} \\
x_{1 d}= & \frac{a_{4}}{a_{3}} \beta+\frac{1}{a_{5}} v_{3}
\end{aligned}
$$

so that

$$
\dot{e}=\left(A_{0}+A_{1}-K\right) e+R(t, \tilde{z})+\left[\begin{array}{l}
v_{1} \\
v_{2} \\
v_{3} \\
v_{4}
\end{array}\right],
$$

where

$$
R(t, \tilde{z})=\left[\begin{array}{c}
\left(a_{2}+a_{5}\right) \tilde{x}_{3}+a_{3} x_{5} \tilde{x}_{4}-\left(\dot{x}_{1 d}-\dot{x}_{1 d}\right) \\
-a_{3} x_{5} \tilde{x}_{3}+\left(a_{2}+a_{5}\right) \tilde{x}_{4}-\left(\dot{x}_{2 d}-\dot{x}_{2 d}\right) \\
0 \\
0
\end{array}\right] .
$$

$K=\operatorname{diag}\left[k_{2}, k_{3}, 0,0\right]$

with $k_{2}, k_{3}$ being feedback control gains, and $v_{i}, i=1,2,3,4$, are extra control terms used for cancelling the coupling terms in the subsequent closed loop stability analyois. 


\subsubsection{Observer Design}

In order to estimate the flux signals, we design a nonlinear observer as follows :

$$
\begin{aligned}
& \dot{x}_{3}=-a_{4} \hat{x}_{3}+a_{5} x_{1}+\left(u_{3}-p x_{5}\right) \hat{x}_{4}+\xi_{11}+\xi_{21} \\
& \dot{x}_{4}=-a_{4} \hat{x}_{4}+a_{5} x_{2}-\left(u_{3}-p x_{5}\right) \dot{x}_{3}+\xi_{12}+\xi_{22}
\end{aligned}
$$

where $\xi_{i j}, 1 \leq i, j \leq 2$, are some suitable functions to be specified in the following. Then, the estimation errors satisfy

$$
\dot{\tilde{z}}=-a_{4} I_{2} \tilde{z}-\left(u_{3}-p x_{5}\right) J_{2} \tilde{z}+E,
$$

where

$$
\begin{aligned}
& I_{2} \triangleq \operatorname{diag}[1,1], \\
& J_{2} \triangleq\left[\begin{array}{cc}
0 & -1 \\
1 & 0
\end{array}\right], \\
& E \triangleq-\left[\begin{array}{l}
\xi_{11}+\xi_{21} \\
\xi_{12}+\xi_{22}
\end{array}\right] .
\end{aligned}
$$

Here, $\xi_{21}, \xi_{22}$ are chosen for the purpose of canceling the coupling terms in equation (23), namely, $R(t, \tilde{z})$, whereas $\xi_{11}, \xi_{12}$ are designed for canceling the coupling terms in the overall closed-loop stability analysis.

For simplicity, we first define some notations in the following:

$$
\begin{aligned}
v_{3} & \triangleq-k_{T} x_{2 d} e_{5} \\
\eta_{0} & \triangleq \frac{k_{T}}{J}\left(\mu_{1}+D+2 \mu_{2} x_{5}-k_{1}\right) x_{2} \\
\eta_{1} & \triangleq \frac{k_{T}}{J}\left(\mu_{1}+D+2 \mu_{2} x_{5}-k_{1}\right) x_{1} \\
\eta_{2} & \triangleq \frac{k_{T}}{J} x_{2} \\
\eta_{3} & \triangleq \frac{k_{T}}{J} x_{1} \\
T_{A} & \triangleq J \tilde{x}_{5 d}+k_{1} \dot{x}_{3 d}+\frac{1}{J}\left(\mu_{1}+D+2 \mu_{2} x_{5}-k_{1}\right)\left(-D x_{5}-T_{L}\right) \\
T_{B} & \triangleq \eta_{0} x_{3}-\eta_{1} x_{4} \\
T_{C} & \triangleq \frac{1}{J}\left(-D x_{5}-T_{2}\right)-\dot{x}_{5 d} \\
T_{D} & \triangleq \eta_{2} x_{3}-\eta_{3} x_{4} \\
\dot{x}_{1 d} & \triangleq-\frac{1}{a_{5} \beta}\left[e_{5} T_{A}+T_{d} T_{C}+\left(e_{5} \eta_{0}+T_{d} \eta_{2}\right) \dot{x}_{3}-\left(e_{5} \eta_{1}+T_{d} \eta_{3}\right) \dot{x}_{4}\right] \\
\dot{x}_{2 d} & \triangleq \frac{T_{A}+\eta_{0} \dot{x}_{3}-\eta_{1} \hat{x}_{4}}{k_{T} \beta} \\
f_{0} & \triangleq a_{2}+a_{5}+\frac{e_{5} \eta_{0}+T_{d} \eta_{2}}{a_{5} \beta} \\
f_{1} & \triangleq-\left(a_{3} x_{5}+\frac{1}{k_{T} \beta} \eta_{0}-k_{T} e_{5}\right) \\
f_{2} & \triangleq a_{3} x_{5}-k_{T} e_{3}-\frac{a_{3} \eta_{1}+T_{d} \eta_{3}}{a_{5} \beta} \\
f_{3} & \triangleq a_{2}+a_{5}+\frac{1}{k_{T} \beta} \eta_{1}
\end{aligned}
$$

Theorem 1 Consider an induction motor whose dynamics are governed by equations (1)-(6) under the assumptions $(A 1)-(A S)$. Then, the control objective can be achieved provided the observer-based controller is designed acconding to (18)-(21) with

$$
\begin{aligned}
& v_{1}=k_{T} \hat{x}_{4} e_{S} \\
& v_{2}=-k_{T} \hat{x}_{3} e_{5} \\
& v_{4}=k_{T} x_{1 d} e_{5}
\end{aligned}
$$

where $\hat{x}_{3}, \hat{x}_{4}$ are obtained from the following nonlinear abserver

$$
\dot{\hat{x}}_{3}=-a_{4} \dot{x}_{3}+a_{5} x_{1}+\left(u_{3}-p x_{5}\right) \dot{x}_{4}+f_{0} e_{1}+f_{1} e_{2}
$$$$
\dot{x}_{4}=-a_{4} \hat{x}_{4}+a_{5} x_{2}-\left(u_{3}-p x_{5}\right) \hat{x}_{3}+f_{2} e_{1}+f_{3} e_{2}
$$

Proof: Choose the Lyapounov function as

$$
V=\frac{1}{2}\left(e^{T} e+\tilde{z}^{T} \tilde{z}+J e_{s}^{2}\right)
$$

From equations $(16)(22)(27)$, the derivative of $\mathrm{V}$ is obtained as follows :

$$
\begin{aligned}
\dot{V}= & e^{T}\left(A_{0}+K\right) e+\dot{z}^{T}\left(-a_{4} I_{2}\right) \tilde{z}-k_{1} e_{3}^{2}+e^{T} R\left(t_{1} \tilde{x}\right)+e_{1} v_{1}+e_{2} v_{2} \\
& +e_{3} v_{3}+e_{4} v_{4}-\left(\xi_{11}+\xi_{21}\right) \tilde{x}_{3}-\left(\xi_{12}+\xi_{23}\right) \tilde{x}_{4}+e_{S}\left(T_{c}-T_{d}\right)
\end{aligned}
$$

First of all,

$$
e_{5}\left(T_{e}-T_{d}\right)=k_{T} e_{5} x_{3} e_{2}-k_{T} e_{5} x_{4} e_{2}+k_{T} e_{3}\left(e_{3} x_{2 d}-e_{4} x_{2 d}\right) .
$$

Next, let

which then yields that

$$
\begin{aligned}
\dot{V}= & -\alpha_{0}\|e\|^{2}-a_{4}\|\tilde{z}\|^{2}-k_{1} e_{5}^{2}+e_{3} v_{3}+e_{4} v_{4}+k_{T} e_{3}\left(e_{3} x_{2 d}-e_{4} x_{1 d}\right) \\
& +e^{T} R(t, \xi)-\xi_{21} \tilde{x}_{3}-\xi_{22} \tilde{x}_{4},
\end{aligned}
$$

where

Furthermore, by substituting the definitions of $v_{3}$ and $v_{4}$ into (34), we obtain

$$
\dot{V} \leq-\alpha_{0}\|e\|^{2}-a_{4}\|\tilde{z}\|^{2}-k_{1} e_{5}^{2}+e^{T} R(t, \tilde{z})-\xi_{21} \tilde{x}_{3}-\xi_{32} \tilde{x}_{4}
$$

It should be noted that $\dot{T}_{d}$ which needs to be used for implementing $\dot{x}_{2 d}$ and $\dot{x}_{1 d}$ includes $\dot{x}_{5}$. This is the reason why we design $\dot{x}_{2 d}$ and $\dot{x}_{1 d}$ instead of $\dot{x}_{2 d}$ and $\dot{x}_{1 d}$. Accordingly, more detailed expressions for $\dot{x}_{1 d}$ and $\dot{x}_{2 d}$ are derived in the following.

To find $\dot{x}_{1 d}$ and $\dot{x}_{2 d}$, we first derive

$$
\dot{T}_{d}=J \bar{x}_{s d}+k_{1} \dot{x}_{s d}+\left(\mu_{1}+D-k_{1}\right) \dot{x}_{5}+2 \mu_{2} x_{5} \dot{x}_{5}
$$

and then substitute equations (11)-(12) into the above to yield

$$
\dot{T}_{d}=T_{A}+T_{B}
$$

Since

we thus have

$$
\begin{aligned}
\dot{x}_{1 d} & =-\frac{1}{a_{3}^{\beta}}\left(e_{3} T_{A}+e_{5} T_{B}+T_{d} T_{C}+T_{d} T_{D}\right) \\
& =-\frac{1}{a_{5} \beta}\left[e_{5} T_{A}+T_{d} T_{C}+\left(e_{5} \eta_{0}+T_{d} \eta_{2}\right) x_{3}-\left(e_{5} \eta_{1}+T_{d} \eta_{3}\right) x_{4}\right] \\
\dot{x}_{2 d} & =\frac{T_{A}+\eta_{0} x_{3}-\eta_{1} x_{4}}{k_{T} \beta}
\end{aligned}
$$

Note that $\dot{x}_{1 d}$ and $\dot{x}_{2 d}$ are equal to $\dot{x}_{1 d}$ and $\dot{x}_{2 d}$, respectively, except that $x_{3}, x_{4}$ are replaced by $\hat{x}_{3}, \hat{x}_{4}$, then, we can easily find that

$$
\begin{aligned}
& \dot{x}_{1 d}-\dot{x}_{1 d}=-\frac{\left[\left(e_{S} \eta_{0}+T_{d} \eta_{2}\right) \tilde{x}_{3}-\left(e_{5} \eta_{1}+T_{d} \eta_{3}\right) \tilde{x}_{4}\right]}{a_{S} \beta} \\
& \dot{x}_{2 d}-\dot{x}_{2 d}=\frac{1}{k_{T} \beta}\left(\eta_{0} \tilde{x}_{3}-\eta_{1} \tilde{x}_{4}\right)
\end{aligned}
$$

To cancel the term involving $R(t, \tilde{z})$ in equation $(35), \xi_{21}$ and $\xi_{22}$ are, hence, designed as

Finally, we have

$$
\begin{aligned}
& \xi_{21}=e_{1}\left(a_{2}+a_{5}+\frac{e_{5} \eta_{0}+T_{d} \eta_{2}}{a_{5} \beta}\right)-e_{2}\left(a_{3} x_{5}+\frac{1}{k_{T} \beta} \eta_{0}\right) \\
& \xi_{22}=e_{1}\left(a_{5} x_{5}-\frac{e_{5} \eta_{1}+T_{d} \eta_{3}}{a_{5} \beta}\right)+e_{2}\left(a_{2}+a_{5}+\frac{1}{k_{T} \beta} \eta_{1}\right)
\end{aligned}
$$

$$
\dot{V} \leq-\alpha_{0}\|e\|^{2}-a_{4}\|\tilde{z}\|^{2}-k_{1} e_{5}^{2}
$$

which thus concludes our proof by following a standard Lyapunov analysis [23]

\subsection{Application to a Single-Link Manipulator}

Previously, we have proposed a nonlinear observer-based tracking control scheme for induction motors with a known desired function of the rotor speed. However, the proposed scheme can be widely applied to the high performance control of systems using induction motors as actuctors. As an illustrative example, we will apply this scheme to the tracking control of robot manipulators actuated by induction motors. For simplicity, here we will only consider a single-link manipulator as shown in Fig. 2 which is driven by an induction motor.

The modeling of the mechanical part of the maripulator system can be described as:

$$
J \ddot{\theta}+D \dot{\theta}=T_{e}-T_{l},
$$

where

$$
T_{L}=I \ddot{\theta}+B \dot{\theta}+m g l \sin \theta,
$$

so that

$$
(J+I) \vec{\theta}+(B+D) \dot{\theta}+m g l \sin \theta=T_{e} .
$$

Note that $I, m, l$ stand for the moment of inertia, the mass, and the length of the link, respectively, $B, g$ represent the viscous frictional coefficient and gravity constant, respectively, and $\theta$ is the angle of rotation of the link.

Similarly, we design the control input of the first stage as

$T_{d}=(J+I) \dot{\theta}_{d}+(B+D) \dot{\theta}_{d}+m g l \sin \theta-k_{\theta}\left(\theta-\theta_{d}\right)-\left(k_{\omega}-(B+D)\right)\left(\dot{\theta}-\dot{\theta}_{d}\right), \quad$ (42) where $k_{\theta}, k_{\omega}$ are some appropriate constants. Denoting $e_{\theta}=\theta-\theta_{d}, e_{\omega}=$ $\dot{\theta}-\dot{\theta}_{d}$, and $e_{m}^{T}=\left[\begin{array}{ll}e_{\theta} & e_{w}\end{array}\right]$, we then have

$$
\dot{e}_{m}=A_{m} e_{m}+B_{m}\left(T_{e}-T_{d}\right)
$$

where

$$
\begin{aligned}
& A_{m}=\left[\begin{array}{cc}
0 & 1 \\
-\frac{k_{t}}{J+I} & -\frac{k_{u}}{J+I}
\end{array}\right] \\
& B_{m}=\left[\begin{array}{c}
0 \\
\frac{1}{J+I}
\end{array}\right] .
\end{aligned}
$$


In the second stage, the design methodology is the same as has been deacribed earlier. Thus, for any $Q>0$, there exists $P>0$ such that $P^{T} A_{m}+A_{m} P=-Q$.

In the same way, we define some notations in the following :

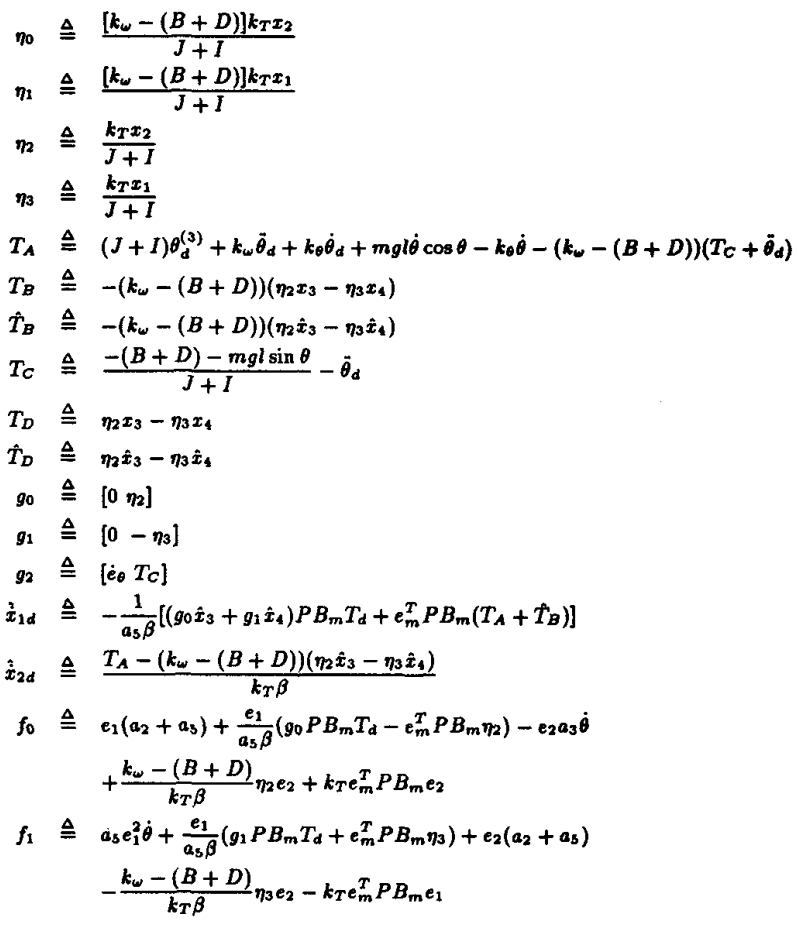

Theorem 2 Consider a single link manipulator actuated by an induction motor whose dynamics are governed by (1) and $(6)$. Let the desired positional trajectory be $\theta_{d}(t)$, whose $\theta_{d}^{(i)}(t), i=1,2,3$ are continuous. Based on the assumptions (AI) and (A2), the objective of position and velocity tracking, i.e.,

$$
\theta(t) \rightarrow \theta_{d}(t), \quad \dot{\theta}(t) \rightarrow \dot{\theta}_{\alpha}(t) \quad \text { exponentially as } t \rightarrow \infty
$$

are achieved provided that the following nonlinear observer-based controller is designed according to (18)-(21) and (42) with

$$
\begin{aligned}
& v_{1}=k_{T} e_{m}^{T} P B_{m} \hat{x}_{4} \\
& v_{2}=-k_{T} e_{m}^{T} P B_{m} \hat{x}_{3} \\
& v_{3}=-k_{T} e_{m}^{T} P B_{m} x_{2 d} \\
& v_{4}=k_{T} e_{m}^{T} P B_{m} x_{1 d}
\end{aligned}
$$

where $\hat{x}_{3}, \hat{x}_{4}$ are estimated by the following nonlinear observer

$$
\begin{aligned}
& \dot{x}_{3}=-a_{4} \dot{x}_{3}+a_{2} x_{1}+\left(u_{3}-p x_{5}\right) \dot{x}_{4}+f_{0} \\
& \dot{\dot{x}_{4}}=-a_{4} \hat{x}_{4}+a_{2} x_{2}-\left(u_{3}-p x_{3}\right) \dot{x}_{3}+f_{1}
\end{aligned}
$$

with

$$
P A_{m}^{T}+A_{m} P=-Q \text {. }
$$

Proof: Choose a Lyaponuov function as

$$
V=\frac{1}{2}\left(e^{T} \dot{e}+\tilde{z}^{T} \tilde{z}+e_{m}^{T} P e_{m}\right) .
$$

From equations (18)(21)(42), the derivative of $\mathrm{V}$ is obtained as follows :

$$
\begin{aligned}
\dot{V}= & e^{T}\left(A_{0}+K\right) e+\tilde{z}^{T}\left(-a_{4} I_{2}\right) \tilde{z}-e_{m}^{T} Q e_{m}+e_{m}^{T} P B_{m}\left(T_{4}-T_{d}\right)+R(t, \tilde{z}) \\
& +e_{1} v_{1}+e_{2} v_{2}+e_{3} v_{3}+e_{4} v_{4}-\left(\xi_{11}+\xi_{21}\right) \tilde{x}_{3}-\left(\xi_{12}+\xi_{22}\right) \tilde{x}_{4}
\end{aligned}
$$

Following the results of Theorem $1, \xi_{11}, \xi_{12}, \xi_{21}$, and $\xi_{22}$ are designed 20

$$
\begin{aligned}
\xi_{11}= & k_{T} e_{m}^{T} P B_{m} e_{2} \\
\xi_{12}= & -k_{T} e_{m}^{T} P B_{m} e_{1} \\
\xi_{21}= & e_{1}\left(a_{2}+a_{s}\right)+\frac{e_{1}}{a_{s} \beta}\left(g_{0} P B_{m} T_{d}-e_{m}^{T} P B_{m} \eta_{2}\right)-e_{2} a_{3} \dot{\theta} \\
& +\frac{k_{\omega}-(B+D)}{k_{T} \beta} \eta_{2} e_{2} \\
\xi_{22}= & a_{5} e_{1}^{2} \dot{\theta}+\frac{e_{1}}{a_{5} \beta}\left(g_{1} P B_{m} T_{d}+e_{m}^{T} P B_{m} \eta_{3}\right)+e_{2}\left(a_{2}+a_{s}\right) \\
& \frac{k_{w}-(B+D)}{k_{T} \beta} \eta_{3} e_{2}
\end{aligned}
$$

So, after some algebraic manipulation, we also have

$$
\dot{V} \leq-\alpha_{0}\|e\|^{2}-a_{4}\|\tilde{z}\|^{2}-e_{m}^{T} Q e_{m} .
$$

which then completes our proof.

\section{Simulation Results}

In this section, the preceding observer-based control scheme is applied to simulation studies of the velocity tracking of the induction motor and the tracking control of a single-link manipulator actuated by an induction motor. The numerical values of the six-pole squirrel-cage induction motor are $R_{s}=3.745 \Omega$, $R_{r}=3.583 \Omega, L_{s}=163.3 \mathrm{mH}, L_{r}=163.3 \mathrm{mH}, M=154.67 \mathrm{mH}, J=0.05 \mathrm{kgm}^{2}$, and $D=0.065 \mathrm{kgm}^{2} \mathrm{~s}^{-1}$.

First the control scheme is tested for load $T_{L}=\mu_{0}+\mu_{1} x_{5}+\mu_{2} x_{5}^{2}$, where $\mu_{0}=3.3, \mu_{1}=0.25, \mu_{2}=0.004$, and the response for sine wave tracking are shown in Fig.3(a)-(b).

Secondly, a single-link manipulator dynamics are used to demonstrate the proposed control law. Here, the numerical values of the manipulator are $I=$ $0.0284 \mathrm{kgm}^{2}, B=0.001 \mathrm{kgm}^{2} \mathrm{~s}^{-1}, m=0.3 \mathrm{~kg}, g=9.8 \mathrm{~m} / \mathrm{s}^{2}$, and $l=0.5 \mathrm{~m}$. The desired trajectory is chosen as $\theta_{d}=\sin 3 t$. Fig.4(a)-(b) show the performance for tracking control of this manipulator.

\section{Experimental Results}

The proposed controller is tested in a system with a squirrel-cage motor rated one house power (HP) with an optical encoder attached to its shaft, a PWM transister inverter, and two personal computers(PC486 with 16 bits $\mathrm{A} / \mathrm{D} \mathrm{D} / \mathrm{A}$ card and PC286) communicating through 8255 cards. The configuration of the system is shown in Fig.5.

\subsection{Rotor position and current detection}

The rotor position is detected by a two pulse (with complementary outputs) optical encoder with two phases set apart by $90^{\circ}$. The resolution is 1024 pulses per revolution. The pulses go through a digital circuit, which accumulates the pulses with 12 bit counter. The corresponding speed is calculated by PC486.

Because of the balanced three phases, i.e., $i_{a s}+i_{b s}+i_{c s}=0$, two currents are adequate for the coordinate transformation. Currents are measured with Hall effect transducers (LB-10GA) and low-pass filters.

\subsection{Control Algorithm Processing}

The control algorithm is performed by $\mathrm{PC486}$. In each period of $2 \mathrm{~ms}$, the program computes the following tasks: rotor position and current sampling, speed calculation, coordinate transformation, control algorithm execution, data fetching to the common memory for PC286, and informing PC286.

\subsection{Results}

The squirrel-cage motor used in the experiment is manufactured by ELMA MOTORs $\mathrm{CO}$. with delta-connected stator. The parameters of the motor are shown below:

\footnotetext{
Rated power $=0.75 \mathrm{~kW}$

Rated current $=4 \mathrm{~A}$

Rated voltage $=220 \mathrm{~V}$

Rated frequency $=60 \mathrm{~Hz}$

Rated speed $=1120 \mathrm{rpm}$

poles $=6$

$R_{\mathbf{a}}=3.745 \Omega$

$R_{r}=3.583 \Omega$

$L_{\Delta}=163.3 \mathrm{mH}$

$L_{\mathrm{r}}=163.3 \mathrm{mH}$

$L_{\mathrm{m}}=154.67 \mathrm{mH}$
}

Fig.6 shows the velocity response for $200 \mathrm{rpm}$

\section{Conclusions}

From the skew-symmetric property of induction motors, the observer-based controller is proposed. Without the measurement of fluxes, velocity and position trackiag can be achieved under the assumptions that the system parameters are known. 
The simulation and experiment results have shown the effectiveness of the proposed controller in combination with the flux observer. The future theoretical work is to enhance the robustness of the controller and/or to add parameter estimation with an adaptive observer.

\section{References}

[1] Paul C. Krause, Analysis of Electric Machinery, McGraw-Hill, 1986.

[2] B. K. Bose, Power Electronics and AC Drives, Prentice-Hall, 1986.

[3] Dong-Il Kim, In-Joong Ha and Myoung-Sam Ko, "Control of Induction Motors via Feedback Linearization with Input-Output Decoupling," Int. J. Control, Vol. 51, No. 4, pp. 863-883, 1990.

[4] Alessandro De Luca and Giovanni Ulivi, "Design of an Exact Nonlinear Controller for Induction Motors," IEEE Trans, on Automatic Control, Vol. 34 No. 12, December 1989.

[5] R. Marino, S. Pereada, P. Valigi, "Adaptive Partial Feedback Linearization of Induction Motors," Proc. 29th CDC, pp. 3313-3318, 1990

[6] R. Marino, S. Pereada, P. Valigi, "Adaptive Input-Output Linearizing Control of Induction Motors," IEEE Trans. Automat. Contr., Vol. 38, No. 2, pp. 208-221, Feb. 1993.

[7] W. Leonhard, "Microcomputer Control of High Dynamic Performance ACDrives-A Survey," Automatica, Vol. 22, No. 1, pp. 1-19, 1986

[8] Allan B. Plunkett, "Direct Flux and Torque Regulation in a PWM InverterInduction Motor Drive," IEEE Trans. on Inductry Applications, Vol. IA-13, No. 2, March/April 1977.

[9] Thomas A. Lipo and Kwong C. Chang, "A New Approach to Flux and Torque-Sensing in Induction Machines," IEEE Trans. on Inductry Applications, Vol. IA-22, No. 4, July/August 1986.

[10] George C. Verghese and Seth R. Sanders, "Observers for Flux Estimation in Induction Machines," IEEE Trans. on Inductrial Electronics, Vol. 35, No. 1 , February 1988.

[11] A. Bellini, G. Figalli and G. Ulivi, "Analysis and Design of a Microcomputerbased Observer for an Induction Machine," Automatica, Vol. 24, No. 4, pp $549-555,1988$.

[12] Rupprecht Gabriel, Werber Leonhard, and Craigj. Nordby, "Field-Oriented Control of a Standard AC Motor Using Mircoprocessors," IEEE Trans. on Inductry Applications, Vol. IA-16, No. 2, March/April 1980.

[13] Zheng Kaung Wu and Elias G. Strangas, "Feed Forward Field Orientation Control of an Induction Motor Using a PWM Voltage Source Inverter and Standardized Single-Board Computers," IEEE Trans. Industrial Electronics, Vol. 35, No. 1, February 1988.

[14] Kenji Kubo, Masahiko Watanabe, Tsiztomu Ohmae, Kenzo Kamiyama, "A Fully Digitalized Speed Regulator using Multimicroprocessor System for Induction Motor Drives," IEEE Trans. on Industry Applications, Vol. IA-21. No. 4, pp. 1001-1008, July/Aug. 1985.

[15] I. Kanellakopoulos, P. T. Krein, and F. Disilvestro, "Nonlinear Flux-Observer Based Control of Induction Motors," Proc. 1992 ACC, pp. 1700-1704.

[16] I. Kanellakopoulos, P. V. Kokotovic, and A. S. Morse, "A Toolkit for Nonlinear Feedback Design," Sytems and Control Letters, Vol. 18, No. 2, pp. 83-92, 1992.

[17] Romeo Ortega, and Carlos Canudas, and Seleme I. Seleme, "Nonlinear Control of Induction Motors Torque Tracking with Unknown Load Disturbance," Proc. 1992 ACC, pp. 206-210.

[18] Jong-Hann Jean and Li-Chen Fu, "A new Adaptive Control Scheme for Induction Motors," 15th National Sympositum on Automatic Control, pp. 593 . 601, Taiwan, R.O.C., 1991.

[19] Ned Mohan, Tore M. Undeland, William P. Robbins, Power Electronics : converters, applications and design, Wiley, 1989.

[20] Joachim Holtz, "Pulsewidth Modulation - A Survey," IEEE Trans. on Industrial Electronics, Vol. 39, No. 5, December, 1992, pp.410-420.

[21] C. M. Liaw, C.T. Pan, and Y. C. Chen, "An Adaptive controller for currentfed induction motor," IEEE Trans. on Aerosp. Elect. Syst., Vol. 24, No. 3 , pp. 250-262, 1988 .

[22] C. C. Chan, W. S. Leung and C. W. Ng, "Adaptive Decoupling Control of Induction Motor Drives, ${ }^{n}$ IEEE Ind. Electron., Vol. IE-37, No. 1, pp. 41-47, Feb. 1990.

[23] M. Vidyasagar, Nonlinear System Analysia, Prentice-Hall, 1992.

[24] I. Kanellakopoulos and P. T. Krein, "Integral-Action Nonlinear Control of Induction Motors," Proc. of IFA C, Vol 7, pp. 251, 1993.
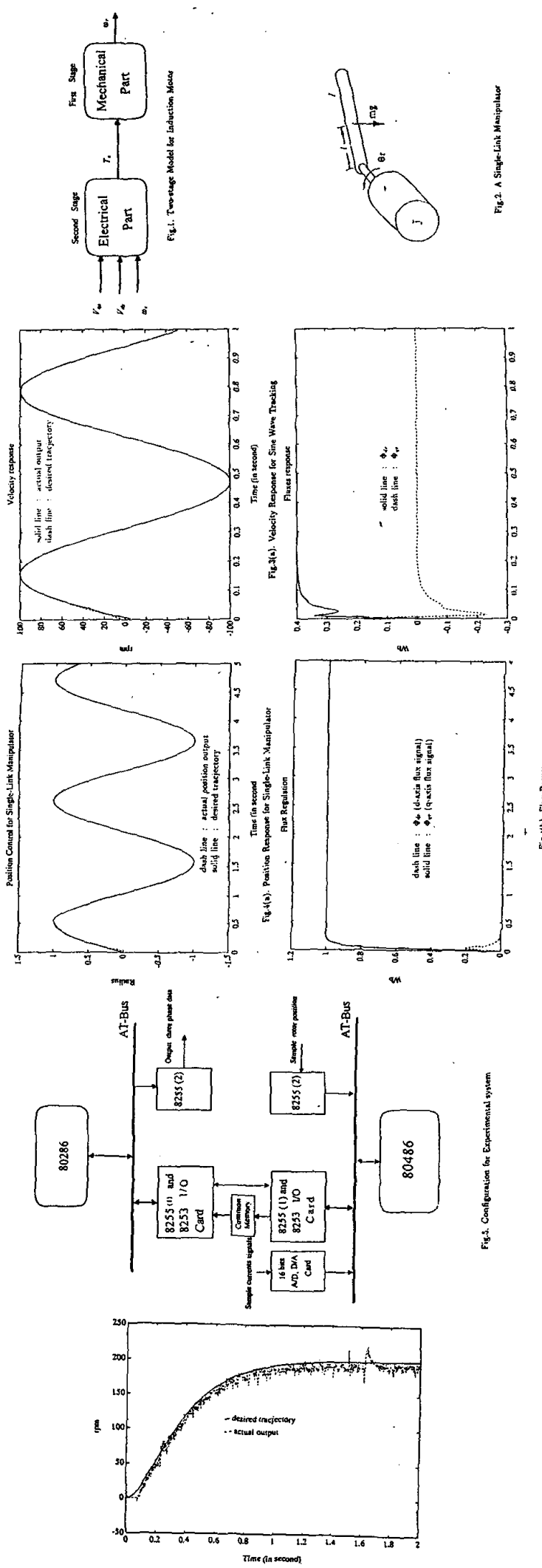

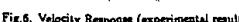

